A N N A L E S Annales de Bretagne et des Pays de l'Ouest

\title{
Histoire du Petit Séminaire de Quintin (1934-1975)
}

\section{Georges Provost}

\section{OpenEdition}

Journals

Édition électronique

URL : http://journals.openedition.org/abpo/3466

DOI : $10.4000 / a b p o .3466$

ISBN : 978-2-7535-5365-1

ISSN : 2108-6443

Éditeur

Presses universitaires de Rennes

Édition imprimée

Date de publication : 30 décembre 2016

Pagination : 203-205

ISBN : 978-2-7535-5363-7

ISSN : 0399-0826

\section{Référence électronique}

Georges Provost, " Histoire du Petit Séminaire de Quintin (1934-1975) », Annales de Bretagne et des Pays de l'Ouest [En ligne], 123-4 | 2016, mis en ligne le 30 décembre 2016, consulté le 23 septembre 2020. URL : http://journals.openedition.org/abpo/3466 ; DOI : https://doi.org/10.4000/abpo.3466 
même s'il ouvre des perspectives de classement qui auraient gagné alors à être mises en œuvre et confrontées aux photographies exposées. Nombre de ces réalisations s'inscrivent, en effet, implicitement ou explicitement, dans les préoccupations de l'Union nationale des Sociétés photographiques de France qui entend constituer, par le biais des sociétés qui la composent, les archives pittoresques des provinces. "Documenter le monde proche " est ainsi, sans conteste, le fil directeur d'une bonne partie des photographies exposées. Conservatoire des traditions, la Bretagne se donne donc ici à voir dans de nombreux clichés, dans tous les sens du terme. Bretonnes en coiffe, scènes de la vie religieuse, activités agricoles et maritimes constituent tout un ensemble de réalisations d'inégal intérêt. On remarque l'influence persistante, du moins jusqu'à la veille du second conflit mondial, du courant pictorialiste qui cherche le dialogue avec la peinture. Les effets de lumière sur les bords de mer, les fumées produites par le brûlage du goémon offrent alors des effets visuels qui s'inscrivent dans ce cadre. Certaines photographies de Georges Nitsch, la plupart du temps réalisées sur des plaques de verre au gélatino-bromure d'argent, se singularisent alors par la qualité du coup d'œil et de la maîtrise technique à l'instar de cette scène de labour (p. 33), prise vers 1930, d'où se dégage une image saisissante du rapport de l'homme à la nature.

Ce souci d'inventaire documentaire se perçoit également dans les nombreuses photographies de la ville de Rennes. Au sein de cet ensemble, là encore très inégal, nombre de réalisations n'offrent qu'un intérêt historique, nullement négligeable du reste. Ainsi en est-il des photographies autour du procès Dreyfus ou du champ de course des Gayeulles, à la charnière des $\mathrm{XIX}^{\mathrm{e}}$ et $\mathrm{xx}^{\mathrm{e}}$ siècles. Les clichés sur les funérailles du cardinal Charost, en novembre 1930, puis ceux sur la construction du quartier Colombier ou du square des Hautes Ourmes dans les années 1970 s'inscrivent également dans cette dimension. On leur préférera les clichés dont l'intérêt documentaire se situe dans la capacité à rendre compte de l'atmosphère d'un moment du quotidien, grâce à une qualité artistique qui ouvre des horizons d'observation et de sensation à l'exemple de la vue stéréoscopique d'Ambroise Poirier prise sur les quais (p. 44), le 17 février 1924, ou de cette scène de noce captée par Georges Dorer à la fin des années 1950 depuis les étages de l'Hôtel de ville (p. 52).

Si le regard de l'amateur ne suscite pas toujours l'adhésion, il n'en reste pas moins que cet ouvrage, dont on peut regretter cependant qu'il n'ait pas pris une forme plus aboutie, permet avec intérêt de replacer la pratique photographique dans le temps et dans l'espace d'une ville de province en soulignant les sociabilités spécifiques qu'elle met en œuvre.

David BENSOUSSAN

Molgno, Yves, Histoire du Petit Séminaire de Quintin (1934-1975), Quintin, Association des Anciens élèves du Petit Séminaire de Quintin, 2012, 353 p.

Quarante ans après l'étude pionnière de Jean-René Chotard sur Guérande (1977), le temps semble opportun d'un retour sur l'histoire des Petits Séminaires bretons qui ont joué un rôle éminent dans la formation des jeunes garçons. Que l'éditeur de cet ouvrage sur le Petit Séminaire de Quintin, soit l'Association des anciens élèves, dont l'auteur est lui-même membre actif, ne saurait être anodin : l'entreprise est largement le fruit d'une démarche participative qui a permis de rassembler clichés, témoignages personnels (85), bulletins imprimés... et de compenser heureusement la faiblesse des archives provenant de l'institution. L'ouvrage témoigne donc d'abord d'une démarche de mémoire mais il offre aussi l'occasion d'une véritable enquête 
historique sur une institution très illustrative des bonheurs, malheurs et surtout ambiguïtés constitutives d'un mode spécifique de formation destiné à " fabriquer des curés " à partir de garçons très inégalement motivés.

De cette exemplarité, la fondation tardive du Petit Séminaire de Quintin est sans doute la première clé : fondé en 1931, l'établissement témoigne très précisément de la volonté de Pie XI, relayée par les évêques, de contrer la chute des vocations en développant des Petits Séminaires homogènes en ce qu'ils conduisent naturellement au Grand, en décalage avec le modèle en vigueur précédemment d'institutions préparant aux carrières civiles au moins autant qu'au sacerdoce. Dans l'esprit de $\mathrm{M}^{\mathrm{gr}}$ Serrand - qui avait également fait construire le Grand Séminaire de Saint-Brieuc (1924-1927) - le Petit Séminaire de Quintin correspondait aussi à un objectif d'unité dans un diocèse très éclaté où la formation des futurs clercs se partageait jusqu'alors entre SaintJoseph de Lannion (qui avait pris le relais de Tréguier) pour les Trégorrois, NotreDame de Campostal à Rostrenen (qui avait pris la succession de Plouguernével) pour les Hauts-Cornouaillais, les Cordeliers de Dinan pour les Gallos. Quintin, géographiquement central, représentait de ce point de vue un choix rationnel. Les bâtiments neufs qui s'élèvent à partir de 1933 sur la colline de Haute-Folie traduisent une logique qui est à la fois de visibilité et de mise à l'écart du monde, pour mieux préserver et cultiver des vocations que l'on sait fragiles. Très soutenue par l'évêque, la nouvelle institution attire à elle les meilleurs enseignants des collèges religieux du diocèse. La réalité est rapidement en deçà des projets, prévus pour 300 élèves : les bâtiments inachevés (seules deux ailes sur trois prévues sont réalisées) n'accueillent à la rentrée 1937 que 191 petits séminaristes dans des conditions spartiates. Malgré le secours éventuel de bourses diocésaines, le placement d'un garçon y demeure peu envisageable pour nombre de familles rurales. Au cours de "l'époque héroïque des débuts " (1934-1952), marquée notamment par les difficultés de l'occupation allemande, la maison parvient néanmoins à s'enraciner dans son environnement.

La rupture identifiée par l'auteur au début des années 1950 tient à plusieurs évolutions dans la manière de mettre en œuvre l'objectif vocationnel, plus impérieux que jamais à l'heure où la diminution sensible des ordinations sacerdotales laisse déjà craindre une " course à l'abîme " (1953). Le renouvellement passe par des enseignants détenteurs de licences universitaires, par un esprit plus ouvert aux innovations culturelles (théâtre, veillées, projections, éducation physique...) et une pédagogie qui mise sur des " équipes " de trois ou quatre élèves. L'ouvrage met très bien en relief l'incidence des conditions matérielles à compter du jour où, en 1952, les Petits Séminaristes peuvent bénéficier de bourses d'État. Bien des familles nombreuses des campagnes des Côtes-du-Nord saisissent alors l'occasion de placer un ou plusieurs garçons à Quintin. Les effectifs croissent en conséquence : 175 élèves en 1952, 346 en 1961 dont $70 \%$ sont boursiers. Le doublement numérique - servi par une très active politique de recrutement via les " Journées des vocations " dans les paroisses, les " retraites d'enfants " et le relais des vicaires-instituteurs - témoigne de l'» âge d'or du Petit Séminaire " (19521962). Vers 1960, le déséquilibre traditionnel en faveur du pays gallo (jusqu'à $80 \%$ des élèves!) paraît même se réduire. Mais rétrospectivement, les faux-semblants apparaissent comme autant d'évidences. La plus large ouverture de la maison rend nécessairement plus floue la dimension vocationnelle, même si personne ne se l'avoue vraiment alors, qu'il s'agisse du clergé dirigeant la maison, des élèves (dont certains disent clairement aujourd'hui qu'ils n'ont jamais songé à la prêtrise), ou de leurs parents trop heureux de pouvoir leur payer des études, quoi qu'il advienne ensuite. L'histoire des années 1950 semble pouvoir être lue au prisme de ces contradictions : le P. Thomas, directeur en 1954, présente le Petit Séminaire comme un " carrefour " et non un " rail " - il est donc normal d'y accueillir des enfants sur d'autres critères que le désir de la prêtrise - mais il n'est guère d'autre avenir présenté aux élèves que la perspective du 
sacerdoce. Les vacances s'allongent (aux Gras et à la Toussaint à partir de 1955) mais il importe qu'en famille ou en " colo ", la surveillance du recteur ou le maintien de cadres collectifs de piété conserve le petit séminariste dans l'esprit de "sa " vocation.

L'optimisme est de rigueur au seuil des années 1960, à l'heure où s'élèvent enfin les murs de la chapelle définitive. Quand les élèves en creusent les fondations, au printemps 1957, la Voix du Petit Séminaire envisage l'avenir sans inquiétude : " J'étais là quand on a commencé, diront bon nombre de recteurs dans cinquante ans! " On le devine : très peu de ces élèves sont devenus prêtres et tous ne le sont pas " restés ". Mais en 1957, plus des trois quarts des élèves de terminale du Petit Séminaire vont encore au Grand et chaque promotion d'ordinands compte $60 \%$ d'anciens de Quintin... Comment imaginer alors que la promotion de $6^{\mathrm{e}}$ admise à la rentrée précédente sera la première à ne fournir aucun prêtre? D'autant que la nouvelle chapelle regarde résolument vers l'avenir : ses volumes, dessinés par le dominicain Cocagnac de la revue Art sacré, convergent vers l'autel, anticipant les réformes conciliaires. L'aspiration au changement semble alors irrépressible, tant elle semble correspondre aux désirs de la jeunesse : on pourra méditer, p. 171, sur cette photo de grands élèves posant derrière des barbelés en 1956... À partir de 1962 s'ouvre " le temps difficile des mutations ", que signale la plongée rapide des effectifs. Distincte de la chute des vocations, elle s'explique d'abord par la multiplication des CES pourquoi envoyer, dès lors, les garçons à Quintin? - et la désaffection des familles pour les pensionnats. Mais les responsables de la maison y voient un motif supplémentaire d'ouvrir largement l'établissement sur un monde qui bouge et que l'on veut envisager positivement, loin du " cocon " et de la "serre chaude " désormais récusés. Comme d'autres Petits Séminaires, Quintin s'avère donc très réceptif aux expérimentations nouvelles - du journal des élèves en 1962 à l'autodiscipline après 1968 - en raison inverse de la rigidité du modèle initial.

À partir de 1966, sous la direction du P. Colleter, la dimension vocationnelle devient de facto périphérique : "séminaire de jeunes " en 1966 puis " centre JeanXXIII " deux ans plus tard, Quintin devient surtout un collège-lycée catholique rayonnant avec succès sur le secteur alentour. Les contrats d'association avec l'État (1968 et 1970), l'accueil d'externes et la mixité (1971), la fusion avec les deux autres écoles catholiques de Quintin (1975), la laïcisation du corps enseignant (un directeur laïc en 1979) jalonnent les étapes d'une reconversion d'autant plus réussie qu'elle est rare parmi les anciens Petits Séminaires bretons (le lycée-collège de Sainte-Anne d'Auray offrirait cependant un autre exemple). Sans doute le lecteur aurait-il aimé que l'histoire retracée par Yves Moigno ouvre davantage de points de comparaison mais la bibliographie sur le sujet et le récent colloque consacré au Petit Séminaire de Sainte-Anne d'Auray (octobre 2015) permettront ici d'utiles extensions. Riche de témoignages sans apprêt, fort de données statistiques patiemment réunies et analysées, nourri des questions de la " génération de la rupture " avec ce mélange de distance et d'empathie qu'autorise le recul du temps, l'ouvrage d'Yves Moigno va, à l'évidence, bien au-delà des seuls anciens élèves.

Georges Provost

Le Page, Dominique (dir.), 11 batailles qui ont fait la Bretagne, Morlaix, Skol Vreizh, 2015,359 p.

L'ouvrage collectif dirigé par Dominique Le Page couvre, à travers les faits de guerre qui ont touché la région, deux millénaires d'histoire de la Bretagne, de 